\title{
Nonlinear Whirlpools Versus Harmonic Waves in a Rotating Column of Stratified Fluid
}

\author{
N. H. Ibragimov ${ }^{1}$, R. N. Ibragimov ${ }^{2 *}$ \\ ${ }^{1}$ Department of Mathematics and Science, Blekinge Institute of Technology, \\ SE-371 79 Karlskrona, Sweden and Research Laboratory \\ "Group analysis of mathematical models in natural sciences, technics and technology" \\ Ufa State Aviation Technical University, 450000 Ufa, Russia \\ ${ }^{2}$ Department of Mathematics University of Texas at Brownsville, TX 78520, USA
}

\begin{abstract}
Propagation of nonlinear baroclinic Kelvin waves in a rotating column of uniformly stratified fluid under the Boussinesq approximation is investigated. The model is constrained by the Kelvin's conjecture saying that the velocity component normal to the interface between rotating fluid and surrounding medium (e.g. a seashore) is possibly zero everywhere in the domain of fluid motion, not only at the boundary. Three classes of distinctly different exact solutions for the nonlinear model are obtained. The obtained solutions are associated with symmetries of the Boussinesq model. It is shown that one class of the obtained solutions can be visualized as rotating whirlpools along which the pressure deviation from the mean state is zero, is positive inside and negative outside of the whirlpools. The angular velocity is zero at the center of the whirlpools and it is monotonically increasing function of radius of the whirlpools.
\end{abstract}

Keywords and phrases: stratified rotating fluids, nonlinear Boussinesq model, exact solutions

Mathematics Subject Classification: 35A20

\section{Introduction}

The Kelvin wave is a large-scale wave motion of great practical importance in the Earth's atmosphere and ocean. The existence of the Kelvin wave relies on a gravity and stable stratification for sustaining a gravitational oscillation, significant Coriolis acceleration, and the presence of vertical boundaries or the equator. Atmospheric Kelvin waves play an important role in the adjustment of the tropical atmosphere to convective latent heat release (see e.g. [16]), in the stratospheric quasibiennial oscillaiton, and in the generation and maintance of the Madden-Julian Oscillation ([11], [9], [22]). Oceanic Kelvin waves play a critical role in tidal motion, in the adjustment of the tropical ocean to wind stress forcing, and in generating and sustaining the El Niño Southern Oscillaton (see e.g [13], [14]). For example, the El Niño thermocline signal in the ocean is found to be propagating up to the west coast of North America as a Kelvin wave [12]. Oceanic internal Kelvin waves have been found to form an important part of how the ocean evolves in response to climate signals [15].

${ }^{*}$ Corresponding author. E-mail: Ranis. Ibragimov@utb.edu 
Internal coastal Kelvin waves can be generated by wind-induced, time-dependent coastal upwelling. Coastal upwelling (downelling) is cased by an Ekman mass flux transported offshore (onshore) and forced by longshore winds. The disturbances can then propagate along the coast as boundary-trapped internal Kelvin waves. The internal Kelvin wave speed depends on the density difference across the interface and is normally much slower than that of surface Kelvin waves. In the ocean, the typical speed for internal coastal Kelvin waves is of order of $1 \mathrm{~m} \mathrm{~s}^{-1}$ and the Rossby radius of deformation is of the order of $10 \mathrm{~km}$ in the latitudes.

In this paper, we address the nonlinear equations of motion for internal waves affected by the earth's rotation. The axes are $x$ (assumed eastward for definiteness) $y$ (northward) and $\widehat{k}$ is the unit vector with $z$ in the vertical direction (opposite gravity). The fluid velocity is $\vec{u}=(u, v, w)$ relative to the Cartesian coordinate system $(x, y, z)$. Motion is considered to be outside frictional boundary layers, allowing viscosity and diffusion to be neglected. In the theory of ocean circulation and within meteorological applications, it is commonplace to make the Boussinesq approximation in which the full variation of density is retained only in the buoyancy force in the vertical momentum balance. Wherever else it occurs, in the horizontal momentum balance, and in the continuity equation, density is replaced by a constant (in the simplest implementation). In particular, this means that the three-dimensional velocity field is assumed to be solenoidal (see e.g. [21]). Thus within the Boussinesq approximation, the governing equations of motion for internal waves, observed in a system of coordinates rotating with angular velocity $\vec{\Omega}$ are written in the form (see e.g., [4])

$$
\begin{aligned}
\rho_{0}\left[\frac{\partial \vec{u}}{\partial t}+\vec{u} \cdot \nabla \vec{u}+2 \vec{\Omega} \times \vec{u}\right] & =-\nabla p-g \rho \widehat{k}, \\
\frac{\partial \rho}{\partial t}+\vec{u} \cdot \nabla \rho+w \frac{d \bar{\rho}}{d z} & =0 \\
\nabla \cdot \vec{u} & =0
\end{aligned}
$$

where $g$ is the acceleration due to gravity so that $p$ and $\rho$ are to be interpreted as the pressure and density departures from their mean state

$$
\bar{\rho}(z)=-\frac{\rho_{0}}{g} N^{2} z, \quad \bar{p}(z)=p_{0}-\rho_{0} g z-g \int_{0}^{z} \bar{\rho}(\xi) d \xi,
$$

in which $\rho_{0}$ is the constant reference density, $\bar{\rho}(z)$ is a background stable density profile with the associated buoyancy frequency $N$ defined by

$$
N^{2}=-\frac{g}{\rho_{0}} \frac{d \bar{\rho}}{d z}
$$

and we require $\rho_{0}+\bar{\rho}$ and $\bar{p}$ to be consistent with the state of rest, i.e.,

$$
\frac{d \bar{p}}{d z}=-\left(\rho_{0}+\bar{\rho}\right) g
$$

The traditional $f$-plane approximation is made whereby we take $2 \vec{\Omega}=(0,0, f)$, where $f$ is the inertial frequency which depends on the rotation rate of the earth (angular velocity $\Omega=2 \pi \mathrm{rad} /$ day $\approx 0.73 \times 10^{-4}$ $\mathrm{s}^{-1}$ ). We derive the exact solutions for the nonlinear model (1.1) - (1.3) in a cylindrical wave field under the Kelvin's hypothesis saying that the component of the velocity that is normal to the lateral circular boundary be zero throughout the domain. The exact solutions will be obtained as invariant solutions associated with the symmetries of the Boussinesq model (1.1) - (1.3).

\section{Modeling Equations}

For completeness, we write explicitly the three-dimensional nonlinear Euler equations of motion (1.1) (1.3) in the cylindrical coordinates within the Boussinesq approximation: 


$$
\begin{aligned}
\frac{\partial u_{r}}{\partial t}+u_{r} \frac{\partial u_{r}}{\partial r}+\frac{u_{\theta}}{r} \frac{\partial u_{r}}{\partial \theta}+w \frac{\partial u_{r}}{\partial z}-\frac{u_{\theta}^{2}}{r}-f u_{\theta} & =-\frac{\partial p}{\partial r} \\
\frac{\partial u_{\theta}}{\partial t}+u_{r} \frac{\partial u_{\theta}}{\partial r}+\frac{u_{\theta}}{r} \frac{\partial u_{\theta}}{\partial \theta}+w \frac{\partial u_{\theta}}{\partial z}+\frac{u_{r} u_{\theta}}{r}+f u_{r} & =-\frac{1}{r} \frac{\partial p}{\partial \theta} \\
\frac{\partial w}{\partial t}+u_{r} \frac{\partial w}{\partial r}+\frac{u_{\theta}}{r} \frac{\partial w}{\partial \theta}+w \frac{\partial w}{\partial z} & =-\frac{\partial p}{\partial z}-\rho g \\
\frac{\partial \rho}{\partial t}+u_{r} \frac{\partial \rho}{\partial r}+\frac{u_{\theta}}{r} \frac{\partial \rho}{\partial \theta} & =\frac{N^{2}}{g} w \\
\frac{1}{r} \frac{\partial\left(r u_{r}\right)}{\partial r}+\frac{1}{r} \frac{\partial u_{\theta}}{\partial \theta}+\frac{\partial w}{\partial z} & =0
\end{aligned}
$$

As he recounted in his presentation to the Royal Society of Edinburgh in 1879, Lord Kelvin thought that the vanishing of the velocity component normal to the wall suggested the possibility that it be zero everywhere. Following this hypothesis, we allow the component of the velocity that is normal to the lateral circular boundary be zero, i.e. we let, in anticipation,

$$
u_{r}=0
$$

throughout the domain and investigate the consequences. The problem which forms the main focus of interest here is to obtain exact solutions of the nonlinear Boussinesq model (2.1) - (2.5) within the Kelvin's hypothesis (2.6):

$$
\begin{aligned}
& \frac{\partial u_{\theta}}{\partial t}+\frac{u_{\theta}}{r} \frac{\partial u_{\theta}}{\partial \theta}+w \frac{\partial u_{\theta}}{\partial z}+\frac{1}{r} \frac{\partial p}{\partial \theta}=0, \\
& \frac{\partial w}{\partial t}+\frac{u_{\theta}}{r} \frac{\partial w}{\partial \theta}+w \frac{\partial w}{\partial z}+\frac{\partial p}{\partial z}+\rho g=0, \\
& \frac{\partial p}{\partial r}-\frac{u_{\theta}^{2}}{r}-f u_{\theta}=0, \\
& \frac{\partial \rho}{\partial t}+\frac{u_{\theta}}{r} \frac{\partial \rho}{\partial \theta}-\frac{N^{2}}{g} w=0, \\
& \frac{1}{r} \frac{\partial u_{\theta}}{\partial \theta}+\frac{\partial w}{\partial z}=0 .
\end{aligned}
$$

Our model is idealized by assuming $N$ to be uniform over the extent of the fluid which corresponds to a vertically linear density variation. While this simplification is commonly used in laboratory and theoretical studies and it is quite reasonable for the thermocline region, it is not common in the deep region of the ocean with the except when considering short wavelengths in comparisons with the scale of density changes [20]. At low frequencies, close to $f$, rotational effects are important. Such internal waves are sometimes called inertial-internal waves. At high frequencies, close to $N$ and far from $f$, rotational effects are negligible.

\section{Preliminaries}

This section provides a concise introduction to the calculus of Lie group analysis which represents a simplified version of the overview of basic concepts of Lie symmetry groups outlined in Ibragimov $\mathcal{E}$ Ibragimov, [8].

Let $x=\left(x^{1}, \ldots, x^{n}\right)$ and $u=\left(u^{1}, \ldots, u^{m}\right)$ be independent and dependent variables, respectively. We will deal with one-parameter groups $G$ of transformations of the form

$$
\begin{array}{ll}
\bar{x}^{i}=\varphi^{i}(x, a), & \left.\varphi^{i}\right|_{a=0}=x^{i}, \\
\bar{u}^{\alpha}=\psi^{\alpha}(x, u, a), & \left.\psi^{\alpha}\right|_{a=0}=u^{\alpha},
\end{array}
$$


depending on a continuous parameter $a$. The infinitesimal transformations of the group $G$ are written

$$
\bar{x}^{i} \approx x^{i}+a \xi^{i}(x), \quad \bar{u}^{\alpha} \approx u^{\alpha}+a \eta^{\alpha}(x, u) .
$$

The generator (which is also sometimes called "symmetry") of the group $G$ is the linear first-order differential operator

$$
X=\xi^{i}(x) \frac{\partial}{\partial x^{i}}+\eta^{\alpha}(x, u) \frac{\partial}{\partial u^{\alpha}} .
$$

We employ the usual convention on summation over repeated indices.

Let us consider a (linear or nonlinear) system of partial differential equations

$$
F_{\alpha}\left(x, u, u_{(1)}, \ldots, u_{(s)}\right)=0, \quad \alpha=1, \ldots, m,
$$

where $u_{(1)}, \ldots, u_{(s)}$ denote the sets of partial derivatives of the respective orders, e.g. $u_{(1)}=\left\{u_{i}^{\alpha}\right\}, u_{(2)}=$ $\left\{u_{i j}^{\alpha}\right\}$ with

$$
u_{i}^{\alpha}=\frac{\partial u^{\alpha}(x)}{\partial x^{i}}, \quad u_{i j}^{\alpha}=\frac{\partial^{2} u^{\alpha}(x)}{\partial x^{i} \partial x^{i}}
$$

The group $G$ is called a symmetry group (or admitted group) for Eqs. (3.3) if the system (3.3) has the same form when it is rewritten in the new variables $\bar{x}, \bar{u}$ :

$$
F_{\alpha}\left(\bar{x}, \bar{u}, \bar{u}_{(1)}, \ldots, \bar{u}_{(s)}\right)=0, \quad \alpha=1, \ldots, m .
$$

The generator of the symmetry group $G$ is called an admitted operator or an infinitesimal symmetry for Eqs. (3.3). The effective way of constructing the symmetry group for Eqs. (3.3) is to solve the determining equations

$$
\left.X_{(s)} F_{\alpha}\left(x, u, u_{(1), \ldots, u_{(s)}}\right)\right|_{(3.3)}=0, \quad \alpha=1, \ldots, m,
$$

for the generator (3.2) of the symmetry group $G$, where $X_{(s)}$ is the $s$ th prolongation for the generator $X$ of the group $G$, and the notation $\left.\right|_{(3.3)}$ means evaluated on the frame.

The admitted group $G$ maps any solution of the system (3.3) into a solution of the same system. A solution that is mapped by $G$ onto itself is known as an invariant solution with respect to the group $G$. The invariant solutions for a group with a generator $X$ are obtained as follows. One calculates the invariants $J(x, u)$ of the group $G$ by solving the first-order linear partial differential equation

$$
X(J) \equiv \xi^{i}(x) \frac{\partial J}{\partial x^{i}}+\eta^{\alpha}(x, u) \frac{\partial J}{\partial u^{\alpha}}=0,
$$

or its characteristic system

$$
\frac{d x^{1}}{\xi^{1}(x)}=\cdots=\frac{d x^{n}}{\xi^{n}(x)}=\frac{d u^{1}}{\eta^{1}(x, u)}=\cdots=\frac{d u^{m}}{\eta^{m}(x, u)} .
$$

It is manifest from Eqs. (3.4) that the group has $n+m-1$ functionally independent invariants of the following form:

$$
\lambda_{1}(x), \ldots, \lambda_{n-1}(x), \quad \Phi_{1}(x, u), \ldots, \Phi_{m}(x, u) .
$$

Then we let

$$
\Phi_{\alpha}=\phi_{\alpha}\left(\lambda_{1}, \ldots, \lambda_{n-1}\right),
$$

solve these equations for $u^{1}, \ldots, u^{m}$, substitute the resulting expressions for $u$ into Eqs. (3.3) and obtain a system of partial differential equations for the unknown functions $\phi_{\alpha}\left(\lambda_{1}, \ldots, \lambda_{n-1}\right)$ depending on $n-1$ variables.

If we will require the invariance with respect to a symmetry group with $n-1$ linearly independent generators, then we will have only one invariant of the form $\lambda(x)$, and the system (3.3) will be reduced to ordinary differential equations for unknown functions $\phi_{\alpha}(\lambda)$. 


\section{Invariant solutions}

The symmetries (3.2) of Eqs. (2.7) - (2.11) contain two arbitrary functions, $\varphi(z), \quad \psi(t)$, and are spanned by the following operators:

$$
\begin{aligned}
& X_{1}=\frac{\partial}{\partial t}, \quad X_{2}=\frac{\partial}{\partial \theta}, \quad X_{3}=\frac{\partial}{\partial z}, \quad X_{\varphi}=g \varphi(z) \frac{\partial}{\partial p}-\varphi^{\prime}(z) \frac{\partial}{\partial \rho}, \quad X_{\psi}=\psi(t) \frac{\partial}{\partial p} \\
& X_{4}=r \frac{\partial}{\partial r}+z \frac{\partial}{\partial z}+u_{\theta} \frac{\partial}{\partial u_{\theta}}+w \frac{\partial}{\partial w}+2 p \frac{\partial}{\partial p}+\rho \frac{\partial}{\partial \rho}, \\
& X_{5}=2(f t+2 \theta) \frac{\partial}{\partial \theta}-4 r \frac{\partial}{\partial r}+2 f r \frac{\partial}{\partial u_{\theta}}+f^{2} r^{2} \frac{\partial}{\partial p} .
\end{aligned}
$$

There are no group extensions in the case of the homogeneous fluid $(N=0)$ and in the following physically irrelevant case for the ocean $f=N$ (see e.g. [10]). For example, in the mid/high latitude upper non-homogeneous ocean $N$ is typically one or two orders of magnitude larger than $f$ $\left(N\right.$ varies from $10^{-3}$ to $10^{-2} \mathrm{~s}^{-1}$ while $\left.f \sim 10^{-4} \mathrm{~s}^{-1}\right)$.

The symmetries (4.1) can be used for obtaining exact solutions of the system (2.7) - (2.11) by computing the invariant and partially invariant solutions (see, e.g. [17]). In order to obtain all possible invariant and partially invariant solutions one has to construct optimal systems of subalgebras of the Lie algebra with the basis (4.1). Note that invariant solutions based on three-dimensional subalgebras are of a particular interest because they are described by systems of ordinary differential equations. We will consider several solutions of this type.

\subsection{Internal oscillation solution}

We will first construct the invariant solution based on the subalgebra $L_{2}=\left\langle X_{2}, X_{3}\right\rangle$. Invariance with respect to the translations in $\theta$ and $z$ generated by $X_{2}$ and $X_{3}$, respectively requires that the dependent variables are functions of $t$ and $r$ only. Accordingly, the system (2.7)-(2.11) reduces to the form

$$
\begin{aligned}
\frac{\partial u_{\theta}}{\partial t} & =0, \\
\frac{\partial p}{\partial r} & =\frac{u_{\theta}^{2}}{r}+f u_{\theta}, \\
\frac{\partial w}{\partial t} & =-\rho g, \\
\frac{\partial \rho}{\partial t} & =\frac{N^{2}}{g} w .
\end{aligned}
$$

We remark that in actuality, since the hydrostatic balance is linear, it should be possible to modify the equations in order to eliminate the gravitational term, and use the so called dynamic pressure (see e.g. section 3 in [1]) similarly to the case of homogeneous fluids. In the case of stratified fluids, it is also possible by writing the density as a sum of a term $\bar{\rho}(z)$ which satisfies the hydrostatic balance, and perturbation $\widetilde{\rho}(x, z, t)$. Integration of Eqs. (4.2)-(4.5) gives the invariant solution

$$
\begin{aligned}
u_{\theta} & =U(r), \\
p & =\int\left[\frac{1}{r} U^{2}(r)+f U(r)\right] d r+V\left(t-t_{0}\right), \\
w & =W_{1}(r) \cos N\left(t-t_{0}\right)+W_{2}(r) \sin N\left(t-t_{0}\right), \\
\rho & =\frac{N^{2}}{g}\left[W_{1}(r) \sin N\left(t-t_{0}\right)-W_{2}(r) \cos N\left(t-t_{0}\right)\right]
\end{aligned}
$$

with arbitrary functions $U(r), W_{1}(r), W_{2}(r)$ and $V(t)$ and arbitrary constant $t_{0}$. 
Particularly, by setting $U=V=0, W_{1}(r)$ and $W_{2}(r)$ are constants, we arrive to the well-known internal oscillation solution

$$
u_{\theta}=0, \quad p=0, \quad w=A \cos N\left(t-t_{0}\right), \quad g \rho=A N \sin N\left(t-t_{0}\right),
$$

where $A, t_{0}$ are arbitrary constants.

\subsection{Non-stationary solution}

Let us construct the invariant solutions based on the three-dimensional subalgebra spanned by the operators $X_{3}, X_{4}, X_{5}$ from (4.1). According to the theorem on representation of non-singular invariant manifolds ([17], Section 14.3), the invariant solutions can be represented via invariants $J\left(t, \theta, r, z, u_{\theta}, w, p, \rho\right)$ of the operators $X_{3}, X_{4}, X_{5}$. The invariance under $X_{3}$ requires that $J$ does not depend on $z$. Thus, we have to solve the system

$$
\begin{aligned}
& X_{4}(J) \equiv r \frac{\partial J}{\partial r}+u_{\theta} \frac{\partial J}{\partial u_{\theta}}+w \frac{\partial J}{\partial w}+2 p \frac{\partial J}{\partial p}+\rho \frac{\partial J}{\partial \rho}=0 \\
& X_{5}(J) \equiv 2(f t+2 \theta) \frac{\partial J}{\partial \theta}-4 r \frac{\partial J}{\partial r}+2 f r \frac{\partial J}{\partial u_{\theta}}+f^{2} r^{2} \frac{\partial J}{\partial p}=0
\end{aligned}
$$

for the function $J=J\left(t, \theta, r, u_{\theta}, w, p, \rho\right)$. Integration of the system (4.11) gives the following basis of invariants:

$$
\begin{aligned}
& J_{1}=t, \quad J_{2}=\frac{2 u_{\theta}+f r}{(f t+2 \theta) r}, \quad J_{3}=\frac{w}{(f t+2 \theta) r} \\
& J_{4}=\frac{8 p+f^{2} r^{2}}{(f t+2 \theta)^{2} r^{2}}, \quad J_{5}=\frac{\rho}{(f t+2 \theta) r} .
\end{aligned}
$$

The representation of the invariant solutions via the basic invariants (4.12) is obtained by assuming that $J_{2}, J_{3}, J_{4}, J_{5}$ are unknown functions of $J_{1}$. This yields the following candidates for the invariant solutions:

$$
\begin{array}{ll}
u_{\theta}=-\frac{f r}{2}+(f t+2 \theta) r F(t), & w=(f t+2 \theta) r G(t), \\
\left.p=-\frac{f^{2} r^{2}}{8}+(f t+2 \theta)^{2}\right) r^{2} H(t), & \rho=(f t+2 \theta) r K(t) .
\end{array}
$$

Substituting (4.13) into Eqs. (2.7) - (2.11) and solving the resulting equations for the unknown function $F(t), G(t), H(t), K(t)$ we obtain

$$
\begin{aligned}
& F(t)=0, \quad G(t)=A \cos (N t)+B \sin (N t), \\
& H(t)=0, \quad K(t)=\frac{N}{g}[A \sin (N t)-B \cos (N t)],
\end{aligned}
$$

where $A$ and $B$ are arbitrary constants. Substituting (4.14) in (4.13) we obtain the following nonstationary solution of the system (2.7)-(2.11):

$$
\begin{aligned}
& u_{\theta}=-\frac{f r}{2}, \quad w=(f t+2 \theta) r[A \cos (N t)+B \sin (N t)] \\
& p=-\frac{f^{2} r^{2}}{8}, \quad \rho=\frac{N}{g}(f t+2 \theta) r[A \sin (N t)-B \cos (N t)] .
\end{aligned}
$$




\subsection{Stationary solution}

Let us construct the invariant solutions based on the three-dimensional subalgebra spanned by the operators $X_{1}, X_{3}, X_{4}$ from (4.1). Proceeding as in the above, we obtain the following candidates for the invariant solutions:

$$
u_{\theta}=r F(\theta), \quad w=r G(\theta), \quad p=r^{2} H(\theta), \quad \rho=r K(\theta) .
$$

Substituting (4.16) in Eqs. (2.7) - (2.11) we obtain

$$
\begin{aligned}
& F(\theta)=F_{0}, \quad G(t)=C_{1} \cos (k \theta)+C_{2} \sin (k \theta), \\
& H(t)=\frac{1}{2}\left(F_{0}^{2}+f F_{0}\right), \quad K(t)=\frac{N}{g}\left[C_{1} \sin (k \theta)-C_{2} \cos (k \theta)\right],
\end{aligned}
$$

where $C_{1}, C_{2}$ are arbitrary constants, $F_{0}$ is an arbitrary constant different from zero, and

$$
k=\frac{N}{F_{0}} .
$$

If $F_{0}=0$ the solution collapses to the trivial solution $u_{\theta}=w=p=\rho=0$.

Substitution of (4.17) into Eqs. (2.7) - (2.11) - (4.16) yields the following steady-state solution of the system (2.7)-(2.11):

$$
\begin{array}{ll}
u_{\theta}=F_{0} r, & w=r\left[C_{1} \cos (k \theta)+C_{2} \sin (k \theta)\right], \\
p=\frac{1}{2}\left(F_{0}^{2}+f F_{0}\right) r^{2}, & \rho=\frac{N}{g} r\left[C_{1} \sin (k \theta)-C_{2} \cos (k \theta)\right] .
\end{array}
$$

\subsection{Nonlinear whirlpools}

Constructing as above the invariant solutions based on the three-dimensional subalgebra spanned by the operators $X_{2}, X_{4}, X_{5}$ from (4.1) we obtain the following candidates for the invariant solutions:

$$
u_{\theta}=-\frac{f r}{2}+z F(t), \quad w=z G(t), \quad p=-\frac{f^{2} r^{2}}{8}+z^{2} H(t), \quad \rho=z K(t) .
$$

Substituting these expressions in Eqs. (2.7) - (2.11) we obtain

$$
F(t)=0, \quad G(t)=0, \quad K(t)=k=\text { const. }, \quad H(t)=-\frac{k}{2} g .
$$

Thus, we have the following solution of the system (2.7)-(2.11):

$$
u_{\theta}=-\frac{f r}{2}, \quad w=0, \quad p=-\frac{f^{2} r^{2}}{8}-\frac{k}{2} g z^{2}, \quad \rho=k z,
$$

where $k$ is an arbitrary constant having the dimension $\left[\mathrm{m}^{-1}\right]$. If we take the coordinate system so that $z$ is directed upward then $k$ should be negative. In this coordinate system the pressure deviation $p$ vanishes on the surface of the funnel (whirlpool) with the apex on the bottom of the basin:

$$
r=\frac{2}{f} \sqrt{-g k} z
$$

and is positive inside of the funnel, i.e. under the condition

$$
r<\frac{2}{f} \sqrt{-g k} z .
$$




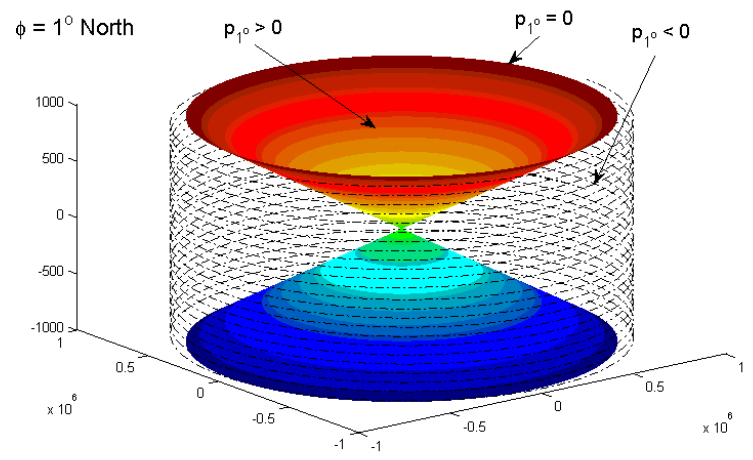

FiguRE 1. Surface of the funel associated with the nonlinear whirlpool in water column of width $1000 \mathrm{~km}$. The choice of the parameter $k=-9.5^{-7}$ is constrained by the requirement of the existence of the lowest latitude whirlpool at latitude $\phi=1^{\circ}$ North.

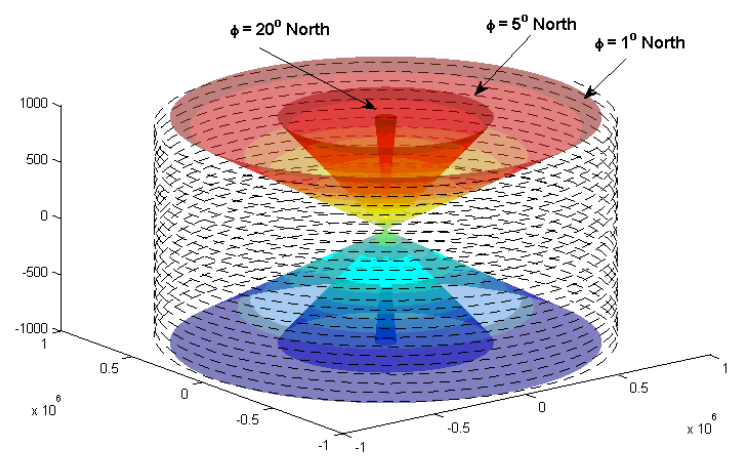

Figure 2. Effects of rotation on the nonlinear whirlpool for different latitudes $\phi=1^{\circ}$, $\phi=5^{\circ}$ and $\phi=20^{\circ}$ North.

This is illustrated in Figure 1 showing the invariant solution (4.19) in the form of the stationary whirlpool expanding/ narrowing with the depth. For this reason, we call the invariant solution (4.19) the whirlpool associated with nonlinear baroclinic Kelvin waves embedded in the rotating column of stratified fluid. The pressure deviation $p$ from the mean state $p_{0}$ is zero on the surface of the whirlpool, positive inside and negative outside of the whirlpool. The intensity of rotation of fluid particles inside the whirlpool is monotonically increasing from zero at the center of the whirlpool to its maximum value of the whirlpool's shell. The existence of the whirlpool in the water column of the fixed radius $r_{0}$ relies on the choice of the parameter $k$. For example, for $r_{0}=1000[\mathrm{~km}]$ and the fixed water column level $H=1[\mathrm{~km}]$ the choice of $k$ satisfying the inequality $|k|<1.653 \times 10^{-7}\left[\mathrm{~m}^{-1}\right]$ guarantees the existence of the whirlpools lying entirely in the cylindrical water column within the latitude range $\phi \in\left[1^{\circ}, 90^{\circ}\right]$ North. Figure 2 is used to compare the intensities of the whirlpools at different latitudes for the given radius $r_{0}=1000[\mathrm{~km}]$ with $k=-9.5^{-7}\left[\mathrm{~m}^{-1}\right]$. We observe that the domain of the positive pressure is narrowing to zero (center of the fluid column) at higher latitudes. 


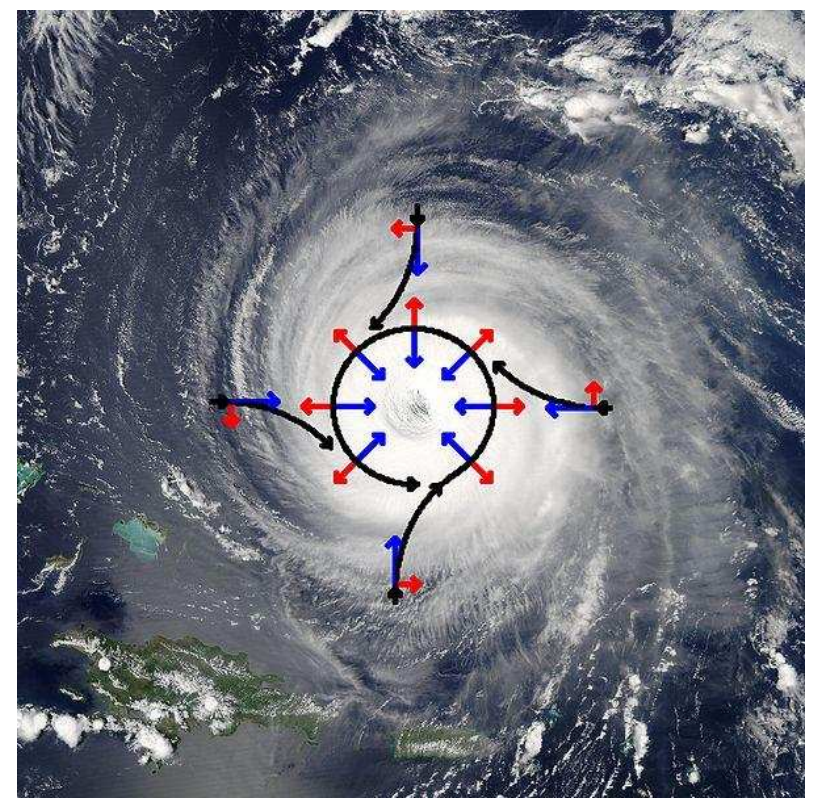

FiguRE 3. Schematic representation of flow around a low-pressure area (in this case, Hurricane Isabel) in the Northern hemisphere. The picture can be found e.g. in http://en.wikipedia.org/wiki/Hurricane_Isabel

\section{Conclusion}

The purpose of this paper was to derive the exact solutions for the nonlinear equations describing the Kelvin waves propagating in cylindrical column of stratified fluid. The effects of the earth's rotation were taken into account. The inquiry is motivated by dynamically significant Coriolis forces in meteorology and oceanographic applications such as nonlinear climate variability models ([7]), the general atmospheric circulation model, weather prediction (see e.g., [3]; [5]; [2];[19]) as well as a variety of applications to largescale nonlinear dispersant operations e.g. Deepwater Horizon incident studied recently in [6]. Three classes of distinctly different exact solutions were obtained by means of group theoretic modelling and so the obtained solutions are invariant solutions associated with the symmetries (4.11)of the Boussinesq model (2.7) - (2.11).

One particular class of invariant solutions can be visualized as rotating whirlpools representing the conic surfaces on which the pressure deviation from the mean state is zero, is positive inside and negative outside of the whirlpools. The angular velocity is zero at the center of the whirlpools and it is monotonically increasing while approaching the boundary of the whirlpools. Such water or atmospheric motion can be associated with hurricanes as illustrated in Figure 3 in which the pressure gradients are represented by blue arrows whereas the Coriolis acceleration, which is always perpendicular to the velocity, by red arrows.

Our model is idealized by assuming $N$ to be uniform over the extent of the fluid. This corresponds to a vertically linear density variation. While this simplification is commonly used in laboratory and theoretical studies and it is quite reasonable for the deep ocean, it is not common in the thermocline region of the ocean except when considering short wavelengths relative to the scale of density changes [20]. An interesting topic for further study could include effects of variable $N$. The results of the forthcoming studies will be reported elsewhere. 
Acknowledgements. The work of Nail H. Ibragimov is partially supported by the Ufa State Aviation Technical University through the contract No. 11.G34.31.0042 in the framework of the resolution No. 220 of the Government of Russian Federation.

\section{References}

[1] A. Ali, H. Kalisch. Mechanical balance laws for Boussinesq models of surface water waves. J. Nonlinear Sci.(2012) 22, 371-498.

[2] S. Balasuriya. Vanishing viscosity in the barotropic $\beta$-plane. J. Math.Anal. (1997) Appl. 214, 128-150.

[3] E. Dewan, R. Picard, R. O'Neil, H. Gardiner, J. Gibson. MSX satellite observations of thunderstorm-generated gravity waves in mid-wave infrared images of the upper stratosphere. Geophys. Res. Lett. (1998) 25, 939-942.

[4] A. Gill. Atmosphere-Ocean Dynamics. New York, etc., Academic Press. (1983)

[5] G.J Haltiner, R.T. Williams. Numerical prediction and dynamic meteorology (1980).

[6] P.A. Hsieh. Application of modflow for oil reservoir simulation during the Deepwater Horizon crisis. Ground Water. (2011) 49 (3), 319-323.

[7] R.N. Ibragimov, N.H. Ibragimov. Effects of rotation on self-resonant internal gravity waves in the ocean. Ocean Modelling, (2010) 31, 80-87.

[8] N.H. Ibragimov, R.N. Ibragimov. Applications of Lie group analysis in Geophysical Fluid Dynamics. Series on Complexity and Chaos, V2, World Scientific Publishers (2011).

[9] N.H. Ibragimov, R.N. Ibragimov. Integration by quadratures of the nonlinear Euler equations modeling atmospheric flows in a thin rotating spherical shell. Phys. Lett. A, (2011) 3858-3865.

[10] N.H. Ibragimov, R.N. Ibragimov. Rotationally symmetric internal gravity waves. Int. J. Non-Linear Mech., (2012) 46-52.

[11] E.D. Maloney, D. L. Hartmann. The Madden-Julian Oscillation, Barotropic Dynamics, and North Pacific Tropical Cyclone Formation. Part I: Observations. J. Atmos. Sci. (2001) 58 (17), 2545-2558.

[12] J.P. McCreary. Eastern tropical ocean response to changing wind systems with applications to El Niño. J. Phys, Oceanogr. (1976) 6, 632-645.

[13] J.P. McCreary. A linear stratified ocean model of the equatorial undercurrent. Phil. Trans. Roy. Soc. London. (1981) $302,385-413$.

[14] J.P. McCreary. Equatorial beams. J. Mar. Res. (1984) 42, 395-430.

[15] D.W. Moore, R.C. Kloosterzeil, W.S. Kessler. Evolution of mixed Rossby gravity waves. J. Geophys. Res. (1998) 103 (C3), 5331-5346.

[16] D. Nethery, D.Shankar. Vertical propagation of baroclinic Kelvin waves along the west coast of India. J. Earth. Syst. Sci. (2007) 116 (4), 331-339.

[17] L.V. Ovsyannikov. Lectures on the theory of group properties of differential equations. Novosibirsk University press, Novosibirsk, 1966. English transl., ed. Ibragimov, N., ALGA Publications, Karlskrona, 2009.

[18] R.D. Romea, J.S. Allen. On vertically propagating coastal Kelvin waves at low latitudes. J. Phys. Oceanogr. (1983) 13 (1),241-1,254.

[19] D.T. Shindell, G.A. Schmidt. Southern Hemisphere climate response to ozone changes and greenhouse gas increases. Res. Lett., (2004) 31, L18209.

[20] C. Staquet, J. Sommeria. Internal Gravity Waves: From instabilities to turbulence. Annu. Rev. Fluid Mech. (2002) 34, 559-593.

[21] R. Szoeke, R.M. Samelson. The duality between the Boussinesq and non-Boussinesq hydrostatic equations of motion. J. Phys. Oceanogr. (2002) 32, 2194-2203.

[22] J.M. Wallace, P.V. Hobbs.Atmospheric Science: An Introductory Survey. Academic Press, (1977) Inc. 76-77. 